\title{
COEFFICIENTS AND INTEGRAL MEANS OF SOME CLASSES OF ANALYTIC FUNCTIONS
}

\author{
T. SHEIL-SMALL
}

\begin{abstract}
The sharp coefficient bounds for the classes $V_{k}$ of functions of bounded boundary rotation are obtained by a short and elementary argument. Elementary methods are also applied for the coefficients of related classes characterised by a generalised Kaplan condition. The result $(1+x z)^{\alpha}(1-z)^{-\beta} \ll(1+z)^{\alpha}(1-z)^{-\beta}$ $(|x|=1, \alpha \geqslant 1, \beta \geqslant 1)$ is proved simply. It is further shown that the functions $(1+z)^{\alpha}(1-z)^{-\beta}$ are extremal for the $p$ th means ( $p$ an arbitrary real) of all Kaplan classes $K(\alpha, \beta)$.
\end{abstract}

1. The Kaplan classes. A function $f(z)=1+a_{1} z+a_{2} z^{2}+\cdots$ analytic and nonzero in $|z|<1$ is said to belong to the Kaplan class $K(\alpha, \beta)(\alpha \geqslant 0, \beta \geqslant 0)$ if for $0<r<1$ and $\theta_{1}<\theta_{2}<\theta_{1}+2 \pi$ we have

$$
-\alpha \pi \leqslant \int_{\theta_{1}}^{\theta_{2}}\left\{\operatorname{Re} \frac{r e^{i \theta} f^{\prime}\left(r e^{i \theta}\right)}{f\left(r e^{i \theta}\right)}-\frac{1}{2}(\alpha-\beta)\right\} d \theta \leqslant \beta \pi .
$$

Notice that each of these inequalities implies the other. This definition includes several well-known classes.

(i) $g(z)=z+\frac{1}{2} a_{1} z^{2}+\cdots$ is close-to-convex of order $\alpha$ iff $g^{\prime} \in K(\alpha, \alpha+2)$.

(ii) $f \in K(\alpha, \alpha)$ iff for a suitable real $\mu$,

$$
\left|\arg \left(e^{i \mu} f(z)\right)\right| \leqslant \alpha \pi / 2 \quad(|z|<1) .
$$

(iii) $g(z)=z+\cdots$ is starlike of $\operatorname{order} \lambda<1$ iff $g(z) / z \in K(0,2(1-\lambda))$.

An alternative definition can be formulated as follows. For $\lambda$ real we write

$$
\Pi_{\lambda}= \begin{cases}K(\lambda, 0) & (\lambda \geqslant 0), \\ K(0,-\lambda) & (\lambda<0),\end{cases}
$$

or, equivalently, $f \in \Pi_{\lambda}$ iff for $|z|<1$,

$$
\operatorname{Re} \frac{z f^{\prime}(z)}{f(z)} \begin{cases}<\frac{1}{2} \lambda & (\lambda>0), \\ >\frac{1}{2} \lambda & (\lambda<0) .\end{cases}
$$

The class $\Pi_{0}=K(0,0)$ consists of the single function $f(z)=1$. We then have

THEOREM A [10]. $f \in K(\alpha, \beta)$ iff we can write $f(z)=g(z) H(z)$, where $g \in \Pi_{\alpha-\beta}$, $\left|\arg \left(e^{i \mu} H\right)\right| \leqslant \frac{1}{2} \pi \min (\alpha, \beta)$ for a suitable real $\mu$.

Received by the editors June 10, 1981.

1980 Mathematics Subject Classification. Primary 30C50, 03C75.

Key words and phrases. Functions of bounded boundary rotation, starlike functions, close-to-convex functions. 
TheOREM B. (a) $0 \leqslant \alpha^{\prime} \leqslant \alpha, 0 \leqslant \beta^{\prime} \leqslant \beta \Rightarrow K\left(\alpha^{\prime}, \beta^{\prime}\right) \subset K(\alpha, \beta)$.

(b) $f \in K(\alpha, \beta) \Leftrightarrow 1 / f \in K(\beta, \alpha)$.

(c) $f \in K(\alpha, \beta) \Leftrightarrow$ for each $p>0, f^{p} \in K(p \alpha, p \beta)$.

(d) $f \in K(\alpha, \beta), g \in K\left(\alpha^{\prime}, \beta^{\prime}\right) \Rightarrow f g \in K\left(\alpha+\alpha^{\prime}, \beta+\beta^{\prime}\right)$.

The functions in $\Pi_{\lambda}$ are characterized by the representation

$$
f(z)=\exp \left(\lambda \int_{T} \log \left(1+e^{i t} z\right) d \mu(t)\right)
$$

for a suitable probability measure on the unit circle $T$. This gives as a dense subclass the $\lambda$-products

$$
f(z)=\prod_{k=1}^{n}\left(1+x_{k} z\right)^{\lambda_{k}}
$$

where $\left|x_{k}\right| \leqslant 1$, sign $\lambda_{k}=\operatorname{sign} \lambda, \sum_{1}^{n} \lambda_{k}=\lambda$. Of special interest are the classes $S(\alpha, \beta)$, where $\alpha \geqslant 0, \beta \geqslant 0$, consisting of functions of the form

$$
f(z)=g(z) / h(z)
$$

where $g \in \Pi_{\alpha}, h \in \Pi_{\beta}$. From Theorem B we see that

$$
S(\alpha, \beta) \subset K(\alpha, \beta),
$$

and if $\alpha>0, \beta>0$, this containment is strict. It is well known that a function $g(z)=z+a_{1} z^{2}+\cdots$ has bounded boundary rotation not exceeding $k \pi$ (the class $V_{k}$ where $\left.k \geqslant 2\right)$ iff $g^{\prime} \in S\left(\frac{1}{2} k-1, \frac{1}{2} k+1\right)$. In particular, such functions $g$ are close-to-convex of order $\frac{1}{2} k-1$ [4].

2. The coefficient problem. The sharp bounds for the coefficients of functions in $V_{k}$ were obtained over two substantial papers [1,4]. The first of these [4] reduced the problem by means of some ingenious extreme point arguments to estimating the coefficients of the special functions $(1+x z)^{\alpha}(1-z)^{-\alpha}$, where $|x|=1, \alpha \geqslant 1$. The estimate

$$
\left(\frac{1+x z}{1-z}\right)^{\alpha} \ll\left(\frac{1+z}{1-z}\right)^{\alpha} \quad(|x|=1, \alpha \geqslant 1)
$$

was obtained with some difficulty in [1] and established the conclusion

$$
f(z) \ll(1+z)^{\alpha} /(1-z)^{\alpha+2}
$$

for $f \in K(\alpha, \alpha+2)(\alpha \geqslant 0)$. Later Brannan [3] simplified the proof of (9) and some similar results, but considerable ingenuity was still required. Even deeper convolution methods, as well as Brannan's results were required to show that

$$
f(z) \ll(1+z)^{\alpha} /(1-z)^{\beta}
$$

for $f \in K(\alpha, \beta)(\alpha \geqslant 1, \beta \geqslant 1)[9,10]$. There is a gap in these results. It is still true that (11) holds when $0<\alpha<1, \beta \geqslant 2-\alpha$. The proof is completely elementary.

THEOREM 1. If $f \in K(\alpha, \beta)$, where $0 \leqslant \alpha \leqslant 1, \beta \geqslant 2-\alpha$, then

$$
f(z) \ll(1+z)^{\alpha} /(1-z)^{\beta} .
$$


Proof. We can write $f=g F$ where $g \in \Pi_{\alpha-\beta}, F \in K(\alpha, \alpha)$. Then

$$
f(z)=\left(F(z) g(z)^{(\beta-1) /(\beta-\alpha)} g(-z)^{(\alpha-1) /(\beta-\alpha)}\right)(g(z) g(-z))^{(1-\alpha) /(\beta-\alpha)} .
$$

Now $g(z)^{(\beta-1) /(\beta-\alpha)} \in K(0, \beta-1)$ and $g(-z)^{(\alpha-1) /(\beta-\alpha)} \in K(1-\alpha, 0)$. Hence

$$
F(z) g(z)^{(\beta-1) /(\beta-\alpha)} g(-z)^{(\alpha-1) /(\beta-\alpha)} \in K(1, \alpha+\beta-1)
$$

and so can be written in the form $H p$, where $H \in K(1,1)$ and $p \in \Pi_{2-\alpha-\beta}$. Standard estimates give $H(z) \ll(1+z)(1-z)^{-1}, p(z) \ll(1-z)^{2-\alpha-\beta}$. Thus

$$
H(z) p(z) \ll(1+z) /(1-z)^{\alpha+\beta-1} \text {. }
$$

Secondly,

$$
(g(z) g(-z))^{(1-\alpha) /(\beta-\alpha)}=k\left(z^{2}\right),
$$

where $k(z) \in \Pi_{\alpha-1}$, which implies

$$
k\left(z^{2}\right) \ll\left(1-z^{2}\right)^{\alpha-1} .
$$

From (13) and (14) we obtain

$$
f(z) \ll \frac{1+z}{(1-z)^{\alpha+\beta-1}}\left(1-z^{2}\right)^{\alpha-1}=\frac{(1+z)^{\alpha}}{(1-z)^{\beta}} .
$$

The solution of the $V_{k}$ problem is an immediate consequence:

Corollary. If $f \in K(\alpha, \beta)$, where $\beta-\alpha \geqslant 2(1-\{\alpha\})$, then (12) holds. In particular, (10) holds.

Proof. If $m=[\alpha]+1=\alpha+1-\{\alpha\}$, we apply the theorem to $f^{1 / m} \in$ $K(\alpha / m, \beta / m)$.

With the help of Theorem 1 we obtain a simple proof of the result of Aharonov and Friedland [1]; also see Brannan [3].

THEOREM 2. For $\alpha \geqslant 1, \beta \geqslant 1$ we have

$$
(1+x z)^{\alpha} /(1-z)^{\beta} \ll(1+z)^{\alpha} /(1-z)^{\beta} \quad(|x| \leqslant 1) .
$$

Proof. Since $(1+x z)^{m} \ll(1+z)^{m}$ for any nonnegative integer $m$, we may assume that $1<\alpha<2, \beta=1$. Put $\alpha=1+\gamma$ and consider

$$
g(z)=(1+x z)^{1+\gamma}(1-z)^{-1} \text {. }
$$

Differentiating gives

$$
g^{\prime}(z)=\frac{(1+x z)^{\gamma}}{(1-z)^{2-\gamma}} \frac{1+(\gamma+1) x-\gamma x z}{(1-z)^{\gamma}} .
$$

By Theorem 1,

$$
(1+x z)^{\gamma} /(1-z)^{2-\gamma} \ll(1+z)^{\gamma} /(1-z)^{2-\gamma} .
$$

It remains to prove

$$
(1+(\gamma+1) x-\gamma x z) /(1-z)^{\gamma} \ll(2+\gamma-\gamma z) /(1-z)^{\gamma},
$$


with the right-hand expression having nonnegative coefficients. The left-hand expression is clearly $\ll 1 /(1-z)^{\gamma}+(\gamma+1-\gamma z) /(1-z)^{\gamma}$ providing that the second term has nonnegative coefficients, which will also show that the right-hand expression in (17) has nonnegative coefficients. The proof is completed by observing that

$$
\frac{d}{d z}\left(\frac{1-\gamma z}{(1-z)^{\gamma}}\right)=\frac{\gamma(1-\gamma) z}{(1-z)^{\gamma+1}}
$$

has nonnegative coefficients for $0<\gamma<1$.

REMARK 1. Although, as we have shown, the coefficient problem for $V_{k}$ can be solved by elementary methods, nevertheless the extreme point methods introduced in [4] seem to be essential for proving (11) in the general case. In view of Theorem 1 it remains an interesting open problem as to whether the functions $(1+x z)^{\alpha}(1-y z)^{-\beta}$ $(|x|=|y|=1)$ represent the extreme points of $K(\alpha, \beta)$ for $0<\alpha<1, \beta \geqslant 2-\alpha$.

The coefficient problem for the remaining values of the parameters $\alpha$ and $\beta$ presents a number of difficulties. In general the function $(1+z)^{\alpha}(1-z)^{-\beta}$ is no longer extremal. The case $\beta=\alpha$ is easily dealt with.

THEOREM 3. If $f \in K(\alpha, \alpha)$ where $0<\alpha<1$, then

$$
\left|a_{n}\right| \leqslant 2 \alpha \quad(n=1,2, \ldots)
$$

This is sharp for $f(z)=\left(1+z^{n}\right)^{\alpha}\left(1-z^{n}\right)^{-\alpha}$.

Proof. Since $f^{1 / \alpha} \in K(1,1)$, we can write

$$
f(z)=\left(\frac{1+x \omega(z)}{1-\omega(z)}\right)^{\alpha} \prec\left(\frac{1+x z}{1-z}\right)^{\alpha}
$$

where $|x|=1$. Since for $0<\alpha<1$ the function $z \rightarrow(1+x z)^{\alpha}(1-z)^{-\alpha}(x \neq-1)$ is convex univalent, we deduce

$$
\left|a_{n}\right| \leqslant \alpha|1+x| \leqslant 2 \alpha \quad(n=1,2, \ldots) .
$$

For the case $\beta=0$ we have

THEOREM 4. If $f \in \Pi_{\alpha}$ where $\alpha>0$, then

$$
\begin{aligned}
& \left|a_{n}\right| \leqslant\left(\begin{array}{l}
\alpha \\
n
\end{array}\right) \quad\left(1 \leqslant n \leqslant\left[\frac{\alpha}{2}\right]+1\right), \\
& \left|a_{n}\right| \leqslant J(\alpha) / n \quad(n>[\alpha / 2]+1)
\end{aligned}
$$

where

$$
J(\alpha)=\left(\left[\frac{\alpha}{2}\right]+1\right)\left(\begin{array}{c}
\alpha \\
{[\alpha / 2]+1}
\end{array}\right) .
$$

In particular, $(1+z)^{\alpha}$ is extremal for the first $[\alpha / 2]+1$ coefficients. Note also that $\left(1+z^{n}\right)^{\alpha / n} \in \Pi_{\alpha}$, so we cannot do better than $\alpha / n$ for the $n$th coefficient.

ProOF. Since $\operatorname{Re}\left(z f^{\prime}(z) / f(z)\right)<\frac{1}{2} \alpha$, we can write

$$
z f^{\prime}(z) / f(z)=\alpha \omega(z) /(1+\omega(z)),
$$


where $\omega(0)=0,|\omega(z)|<1$. We deduce that

$$
\left|\sum_{k=0}^{\infty}(k+1) a_{k+1} z^{k}\right| \leqslant\left|\sum_{k=0}^{\infty}(k-\alpha) a_{k} z^{k}\right| \quad(|z|<1) .
$$

As shown by Clunie [5] this inequality implies

$$
\sum_{k=0}^{n}(k+1)^{2}\left|a_{k+1}\right|^{2} \leqslant \sum_{k=0}^{n}(k-\alpha)^{2}\left|a_{k}\right|^{2} \quad(n=0,1,2, \ldots) .
$$

Hence

$$
(n+1)^{2}\left|a_{n+1}\right|^{2} \leqslant \sum_{k=0}^{n}\left(\alpha^{2}-2 \alpha k\right)\left|a_{k}\right|^{2}
$$

Now equality occurs here when $\omega(z)=z, f(z)=(1+z)^{\alpha}$; hence

$$
(n+1)^{2}\left(\begin{array}{c}
\alpha \\
n+1
\end{array}\right)^{2}=\sum_{k=0}^{n}\left(\alpha^{2}-2 \alpha k\right)\left(\begin{array}{l}
\alpha \\
k
\end{array}\right)^{2} .
$$

Since $a_{0}=1$, we obtain in the case $n=0,\left|a_{1}\right| \leqslant \alpha$. Suppose $n \leqslant \frac{1}{2} \alpha$ and assume we have shown that $\left|a_{k}\right| \leqslant\left(\begin{array}{l}\alpha \\ k\end{array}\right)(1 \leqslant k \leqslant n)$. Then

$$
(n+1)^{2}\left|a_{n+1}\right|^{2} \leqslant \sum_{k=0}^{n}\left(\alpha^{2}-2 \alpha k\right)\left(\begin{array}{l}
\alpha \\
k
\end{array}\right)^{2}=(n+1)^{2}\left(\begin{array}{c}
\alpha \\
n+1
\end{array}\right)^{2},
$$

so $\left|a_{n+1}\right| \leqslant\left(\begin{array}{c}\alpha \\ n+1\end{array}\right)$. By induction this holds up to $n=[\alpha / 2]$. If $n>[\alpha / 2]$, then

$$
(n+1)^{2}\left|a_{n+1}\right|^{2} \leqslant \sum_{k=0}^{[\alpha / 2]}\left(\alpha^{2}-2 \alpha k\right)\left|a_{k}\right|^{2} \leqslant \sum_{k=0}^{[\alpha / 2]}\left(\alpha^{2}-2 \alpha k\right)\left(\begin{array}{l}
\alpha \\
k
\end{array}\right)^{2}=J^{2}(\alpha),
$$

and we obtain (20).

REMARK 2. For $0<\alpha \leqslant 2$ this gives the sharp result

$$
\left|a_{n}\right| \leqslant \alpha / n \quad(n=1,2, \ldots)
$$

obtained by Clunie [5] and Pommerenke [8] in the context of meromorphic starlike functions. It seems unlikely that the $J(\alpha)$ estimate is sharp when $\alpha>2$. A tentative conjecture is that

$$
\left|a_{n}\right| \leqslant \begin{cases}\left(\begin{array}{l}
\alpha \\
n
\end{array}\right) & (1 \leqslant n \leqslant[\alpha]) \\
\alpha / n & (n>[\alpha])\end{cases}
$$

REMARK 3. Although $(1+z)^{\alpha}(1-z)^{-\beta}$ is not extremal for the coefficients for every value of $\alpha$ and $\beta$, we conjecture that the weaker Rogosinski dominance holds:

$$
\sum_{k=1}^{n}\left|a_{k}\right|^{2} \leqslant \sum_{k=1}^{n} A_{k}^{2} \quad(n=1,2, \ldots)
$$

for $f(z)=1+\sum_{1}^{\infty} a_{n} z^{n} \in K(\alpha, \beta)$, where $A_{n}=A_{n}(\alpha, \beta)$ are the coefficients of $(1+z)^{\alpha}(1-z)^{-\beta}$. This is true for $\Pi_{\alpha}(\alpha>0)$ by subordination: if $f \in \Pi_{\alpha}$, then $f(z) \prec(1+z)^{\alpha}$. If this conjecture is true, it implies that for every $\alpha$ and $\beta$, the function $(1+z)^{\alpha}(1-z)^{-\beta}$ is extremal for the $p$ th integral means of $f \in K(\alpha, \beta)$ $(p>0)$. We prove this result in the next section. 


\section{Integral means.}

THEOREM 5. If $f(z) \in K(\alpha, \beta)$, then for each convex function $\Phi$ on $(-\infty, \infty)$, we have, for $0<r<1$,

$$
\int_{-\pi}^{\pi} \Phi\left(\log \left|f\left(r e^{i \theta}\right)\right|\right) d \theta \leqslant \int_{-\pi}^{\pi} \Phi\left(\log \left|k_{\alpha, \beta}\left(r e^{i \theta}\right)\right|\right) d \theta
$$

where $k_{\alpha, \beta}(z)=(1+z)^{\alpha}(1-z)^{-\beta}$.

We follow a method similar to the argument of Leung [7], who dealt with the close-to-convex case $\alpha=1, \beta=3$, making use of Baernstein's star function [2]. The proof is elementary in that no use is made of Baernstein's fundamental result that $u^{*}$ is subharmonic when $u$ is. Instead we require four observations concerning the star function.

LEMMA 1. (a) If $u(z)$ is subharmonic in $|z|<1$ and if $\omega(z)$ is analytic with $\omega(0)=0,|\omega(z)|<1$, then:

$$
\left(u\left(\omega\left(r e^{i \theta}\right)\right)\right)^{*} \leqslant\left(u\left(r e^{i \theta}\right)\right)^{*} \quad(0<r<1,0 \leqslant \theta \leqslant \pi)
$$

(b) if $u$ and $v \in L^{1}(-\pi, \pi)$, then

$$
(u+v)^{*} \leqslant u^{*}+v^{*}
$$

(c) if $u$ and $v$ are even on $[-\pi, \pi]$ and nondecreasing on $[-\pi, 0]$, then

$$
u^{*}+v^{*}=(u+v)^{*}
$$

(d) suppose that $u$ and $v \in L^{1}(-\pi, \pi)$ and

$$
\begin{gathered}
\int_{-\pi}^{\pi} u(t) d t=\int_{-\pi}^{\pi} v(t) d t, \\
u^{*}(\theta) \leqslant v^{*}(\theta) \quad(0 \leqslant \theta \leqslant \pi) ;
\end{gathered}
$$

Then for every convex function $\Phi$ on $(-\infty, \infty)$,

$$
\int_{-\pi}^{\pi} \Phi(u(t)) d t \leqslant \int_{-\pi}^{\pi} \Phi(v(t)) d t
$$

Conversely, (28) implies both (26) and (27).

Proof. (a) follows on an application of Riesz's subordination inequality [6, p. 11]. (b) is trivial. (c) follows from the observation that $w^{*}(\theta)=\int_{-\theta}^{\theta} w(t) d t(0 \leqslant \theta \leqslant \pi)$ for $w(\theta)$ even on $[-\pi, \pi]$ and nondecreasing on $[-\pi, 0]$. To prove (d) we recall that (27) implies (28) for every nondecreasing convex $\Phi$ on $(-\infty, \infty)$. Now it can be shown (exercise) that every convex function on $(-\infty, \infty)$ can be decomposed into the sum of a nondecreasing convex function on $(-\infty, \infty)$ with a nonincreasing convex function on $(-\infty, \infty)$. Therefore we need to show that (28) holds for every nonincreasing convex $\Phi$ on $(-\infty, \infty)$. But then $\Phi(-x)$ is nondecreasing convex and so we require

$$
(-u)^{*}(\theta) \leqslant(-v)^{*}(\theta) \quad(0 \leqslant \theta \leqslant \pi)
$$


Writing $I=[-\pi, \pi]$ we have

$$
\begin{aligned}
(-u)^{*}(\theta) & =\sup _{|E|=2 \theta}\left(\int_{E}-u(t) d t\right)=\sup _{|E|=2 \theta}\left(-\int_{-\pi}^{\pi} u(t) d t+\int_{I-E} u(t) d t\right) \\
& =-\int_{-\pi}^{\pi} u(t) d t+u^{*}(\pi-\theta) \leqslant-\int_{-\pi}^{\pi} v(t) d t+v^{*}(\pi-\theta)=(-v)^{*}(\theta) .
\end{aligned}
$$

Conversely, (28) implies (27) [2] and (28) implies (26) by taking $\Phi(x)=x$ and $\Phi(x)=-x$.

LEMMA 2. If $f \in K(\alpha, \beta)$ we can write

$$
f(z)=\left(1+\omega_{1}(z)\right)^{\alpha} /\left(1-\omega_{2}(z)\right)^{\beta} \quad(|z|<1),
$$

where $\omega_{i}$ are analytic, $\omega_{i}(0)=0$ and $\left|\omega_{i}(z)\right|<1(|z|<1, i=1,2)$ (i.e. $\omega_{i}$ are Schwarz functions).

Proof. By Theorem A we can write $f=g H$ where $g \in \Pi_{\alpha-\beta}$ and $H \in K(\lambda, \lambda)$ $(\lambda=\min (\alpha, \beta))$. It is well known that a function $h \in \Pi_{-2}$ is subordinate to $(1+z)^{-2}$ and, as $g=h^{(\beta-\alpha) / 2}$ for some such $h$ by Theorem $\mathrm{B}(\mathrm{c}), g$ is subordinate to $(1+z)^{\alpha-\beta}$. Also $H=P^{\lambda}$, where $P \in K(1,1)$, and so is subordinate to $(1+x z)(1-z)^{-1}$ for some $x(|x|=1)$. Thus we can write, for suitable Schwarz functions $\sigma_{j}$,

$$
f(z)=\left(\frac{1+\sigma_{1}(z)}{1-\sigma_{2}(z)}\right)^{\lambda}\left(1+\sigma_{3}(z)\right)^{\alpha-\beta} .
$$

It only remains to show that if $\mu>0, \nu>0$, then for Schwarz functions $\tau_{i}$, $\left(1+\tau_{1}\right)^{\mu}\left(1+\tau_{2}\right)^{\nu}$ is subordinate to $(1+z)^{\mu+\nu}$. Clearly we may assume that $\mu+\nu$ $=1$. The result follows on taking $\operatorname{logs}$, since $\log (1+z)$ is convex univalent.

Lemma 3. Suppose that $F(z)=1+A_{1} z+\cdots, G(z)=1+B_{1} z+\cdots$ are analytic and nonzero in $|z|<1$, each having real coefficients with $A_{1}>0, B_{1}>0$, and suppose further that the two functions $z F^{\prime}(z) / F(z), z G^{\prime}(z) / G(z)$ are typically real in $|z|<1$. Then if $f \prec F, g \prec G$, we have, for every convex function $\Phi$ on $(-\infty, \infty)$,

$$
\int_{-\pi}^{\pi} \Phi\left(\log \left|f\left(r e^{i \theta}\right) g\left(r e^{i \theta}\right)\right|\right) d \theta \leqslant \int_{-\pi}^{\pi} \Phi\left(\log \left|F\left(r e^{i \theta}\right) G\left(r e^{i \theta}\right)\right|\right) d \theta .
$$

Proof. By Lemma 1(d) we must prove

$$
\left(\log \left|f\left(r e^{i \theta}\right) g\left(r e^{i \theta}\right)\right|\right)^{*} \leqslant\left(\log \left|F\left(r e^{i \theta}\right) G\left(r e^{i \theta}\right)\right|\right)^{*} .
$$

((26) holds since both integrals are zero.) By Lemma 1(a), (b) the left expression is

$$
\leqslant\left(\log \left|F\left(r e^{i \theta}\right)\right|\right)^{*}+\left(\log \left|G\left(r e^{i \theta}\right)\right|\right)^{*} .
$$

Since $F$ has real coefficients, $\log \left|F\left(r e^{i \theta}\right)\right|$ is even on $[-\pi, \pi]$. Also

$$
\frac{\partial}{\partial \theta} \log \left|F\left(r e^{i \theta}\right)\right|=-\operatorname{Im} \frac{r e^{i \theta} F^{\prime}\left(r e^{i \theta}\right)}{F\left(r e^{i \theta}\right)}
$$

is nonzero and has constant sign for $\theta \in(-\pi, 0)$. Fixing $\theta$ this sign remains constant when $r$ varies (by continuity), and, hence, letting $r \rightarrow 0$, the sign is that of $-A_{1} \sin \theta$, 
i.e. it is positive. Thus $\log \left|F\left(r e^{i \theta}\right)\right|$ is increasing on $[-\pi, 0]$. Similarly for $\log \left|G\left(r e^{i \theta}\right)\right|$. We obtain (32) by applying Lemma 1(c) to (33).

Proof of TheOREM 5. The result follows from Lemmas 2 and 3 by putting $F(z)=(1+z)^{\alpha}, G(z)=(1-z)^{-\beta}$.

\section{REFERENCES}

1. D. Aharonov and S. Friedland, On an inequality connected with the coefficient conjecture for functions of bounded boundary rotation, Ann. Acad. Sci. Fenn. Ser. A I Math. 524 (1972).

2. Albert Baernstein II, Integral means, univalent functions and circular s!̣metrization. Acta Math. 133 (1974), 139-169.

3. D. A. Brannan, On coefficient problems for certain power series (Proc. Sympos. Complex Analysis. Canterbury, 1973), London Math. Soc. Lecture Note Ser., No. 12. Cambridge Univ. Press, London, 1974, pp. 17-27.

4. D. A. Brannan, J. G. Clunie and W. E. Kirwan, On the coefficient prohlem for functions of bounded boundary rotation, Ann. Acad. Sci. Fenn. Ser. A I Math. 523 (1973).

5. J. Clunie, On meromorphic schlicht functions, J. London Math. Soc. 34 (1959). 215-216.

6. Peter L. Duren, Theory of $H^{p}$ spaces, Academic Press, New York. 1970.

7. Y. J. Leung, Integral means of the derivatives of some univalent functions. Bull. London Math. Soc. 11 (1979), 289-294.

8. Ch. Pommerenke, On meromorphic starlike functions, Pacific J. Math. 13 (1963), 221-235.

9. St. Ruscheweyh, Some convexity and convolution theorems for analitic functions, Math. Ann. 238 (1978), 217-228.

10. T. Sheil-Small, The Hadamard product and linear transformations of classes of analytic functions, $\mathrm{J}$. Analyse Math. 34 (1978), 204-239.

Department of Mathematics, University of York, Heslington, York, Y01, 5DD, England 